\title{
Evaluación de dos métodos de análisis dietarios aplicados en la Bandurria Theristicus melanopis (Gmelin 1789) y el Queltehue Vanellus chilensis (Molina 1782)
}

\section{Evaluation of two dietary analysis methods applied in the Black-faced Ibis Theristicus melanopis (Gmelin 1789) and the Southern Lapwing Vanellus chilensis (Molina 1782)}

\author{
Alberto Gantz ${ }^{1 *}$, Jaime R. Rau ${ }^{1}$, Soraya Sade ${ }^{1} \&$ Miguel Yañez ${ }^{2}$ \\ ${ }^{1}$ Laboratorio de Ecología, Departamento de Ciencias Biológicas y Biodiversidad, Universidad de Los Lagos, Osorno, Chile. \\ ${ }^{2}$ Departamento de Estadística, Facultad de Ciencias, Universidad del Bio-Bio, Avenida Collao 1202, Casilla 5-C, Concepción, \\ Chile. \\ *E-mail: agantz@ulagos.cl
}

\begin{abstract}
RESUMEN
Los estudios dietarios contribuyen a comprender la ecología de las aves y son relevantes en programas de conservación de los organismos. Contrastamos los métodos de análisis dietarios fecales y estomacales en la Bandurria y en el Queltehue, para evaluar su sensibilidad para discriminar las presas consumidas. La diversidad y la abundancia de presas detectadas fueron mayores en los estómagos que en las heces en ambas especies de aves. El Queltehue no mostró una asociación en el número de categorías tróficas, ni una similitud en la composición taxonómica de la dieta entre ambos métodos, lo que sugiere que los análisis fecales no son confiables y pueden conducir a interpretaciones erróneas de la ecología trófica de esta especie de ave. La Bandurria mostró una alta asociación en el número de presas entre ambos métodos y la composición taxonómica y abundancia de presas en las heces fue concordante con la detectada en los estómagos, sugiriendo que los análisis fecales entregan resultados confiables y coherentes entre ambos métodos de análisis dietario; se recomienda su uso complementado con métodos moleculares de análisis de contenido estomacal como los isótopos estables si el objetivo de la investigación es desarrollar estudios a largo plazo.
\end{abstract}

Palabras Clave: Alimento, análisis dietario, análisis estomacales, análisis fecales, aves

\begin{abstract}
Dietary studies contribute to our understanding of bird ecology and are an important source of information for programs promoting the conservation of organisms. We contrasted fecal and stomach content methods of dietary analysis in the Black-faced Ibis and the Southern Lapwing, in order to evaluate the degree of sensitivity in distinguishing consumed prey. Diversity and abundance of prey detected were greater in stomach content samples than in fecal samples in both bird species. The Southern Lapwing did not show association in the number of prey, or a similarity in the taxonomic composition of preys between both methods, suggesting a lack of reliability in the fecal analysis that might lead to misunderstanding the trophic ecology of this bird species. The Black-faced Ibis, show a high association in the number of preys between both methods, and the taxonomic composition and abundance of prey in feces were concordant with that detected in the stomach contents, suggesting that the fecal analysis give reliable results which are coherent between both methods of dietary analysis; this method of analysis is, therefore, recommended, and must be complemented with molecular methods of stomach content analysis, such as the stable isotopes if the scopes of the research is to carry out long term studies.
\end{abstract}

KEYwORDs: Birds, dietary analysis, fecal analysis, foods, stomach content analysis

\section{INTRODUCCIÓN}

Los estudios dietarios contribuyen sustancialmente a conocer y comprender la ecología y evolución de las aves y generan información relevante en programas de conservación y manejo de vida silvestre (Brown \& Ewins 1996, Jordan
2005). Se han desarrollado numerosas metodologías para el análisis dietario en aves. Algunas de ellas son simples observaciones directas de las presas consumidas (Harris \& Wanless 1993). Otras contemplan análisis de egagrópilas o regurgitados especialmente aplicados en aves rapaces (Muñoz-Pedreros et al. 2004) y aves marinas (Barret et 
al. 2007), respectivamente. Actualmente, son utilizados procedimientos con técnicas moleculares como la proporción de isótopos estables de carbono y nitrógeno, los análisis de ácidos grasos, la reacción de la cadena de polimerasa (PCR) y la pirosecuenciación del ADN de las presas, entre otros (Bearhop et al. 2004, Barret et al. 2007, Clare et al. 2009). Sin embargo, tradicionalmente se han utilizado en los análisis dietarios métodos directos e indirectos como los análisis estomacales y fecales, respectivamente (Taylor \& O’Halloran 1997, Barrett et al. 2007). Estos análisis son de bajo costo y relativamente simples de desarrollar (Barret et al. 2007). Específicamente, los análisis estomacales presentan la ventaja que se puede evaluar el contenido completo con un buen grado de precisión, pero adolece de la necesidad de sacrificar el animal, lo que genera serias limitaciones éticas en la utilización de este método en particular en los estudios sobre variaciones tróficas a largo plazo (Weiser \& Powell 2011), además de ser impracticable en especies raras y protegidas (Waldner \& Traugot 2012). Alternativamente, los análisis fecales carecen de limitaciones éticas y son bastante útiles en análisis en escalas espaciales y temporales amplias, pero adolecen del nivel de precisión de los análisis estomacales, debido a que los restos de presas se encuentran más disgregados en las heces (Jordan 2005).

En el presente estudio evaluamos la validez de los métodos estomacales y fecales utilizando a la Bandurria (Theristicus melanopis Gemlin 1789) y el Queltehue (Vanellus chilensis Molina 1782) como modelos de estudio, para discriminar el grado de sensibilidad para distinguir las presas consumidas al utilizarse ambos métodos e identificar el más idóneo para análisis dietarios en ambas especies.

\section{MATERIAL Y MÉTODOS}

Analizamos el contenido de 10 estómagos de Queltehues y 10 de Bandurrias colectadas en praderas agrícolas de Chahuilco ( $\left.40^{\circ} 43^{\prime} \mathrm{S}, 72^{\circ} 51^{\prime} \mathrm{O}\right)$, Osorno, sur de Chile, durante Agosto (invierno austral) de 1999. Ambas especies de aves poseen protección total de caza por las leyes chilenas, pero para el presente estudio obtuvimos una autorización especial del Servicio Agrícola y Ganadero (SAG, Departamento DEPROREN, Resolución Acta $\mathrm{N}^{\circ}$ 2490, 17/08/1999). Las aves fueron cazadas al atardecer en las praderas del área de estudio. Los estómagos fueron extraídos a la brevedad e inyectados con AFA (solución de 2 partes de ácido acético, 50 partes de alcohol $96^{\circ}$ y 40 partes de agua destilada) para prevenir digestión post-mortem de las presas (Korschgen 1987, Wobeser et al. 1987). Simultáneamente, en las mismas praderas recolectamos 45 heces de Bandurrias y 34 de Queltehues. Las heces de ambas especies de aves fueron identificadas por la marcada diferencia en su tamaño. Los análisis sólo consideraron heces frescas (blandas al tacto). Las heces fueron guardadas en bolsas de papel y congeladas para su posterior análisis. Éstas fueron lavadas en un tamiz de $0,1 \mathrm{~mm}$ de abertura de malla y depositadas en placas Petri para ser analizadas bajo lupa estereoscópica en el laboratorio. Tanto en los estómagos como en las heces cuantificamos los restos de presas y se identificaron al menor nivel de resolución taxonómico con la ayuda de guías entomológicas (González 1989, Peña 1998) y la colaboración del Laboratorio de Entomología del SAG, Osorno, Chile. Tanto en estómagos como en las heces, contamos todos los restos identificables de élitros, mandíbulas (izquierda y derecha), y cabezas; dividimos el número de élitros y mandíbulas por dos y contamos individualmente las cabezas (Calver \& Wooler 1982). Consideramos dos élitros y dos mandíbulas de lados opuestos y una cabeza, como un individuo.

Comparamos ambos métodos de análisis dietario evaluando la diversidad de las categorías de presas identificadas, con el Índice de Shannon y su varianza analítica con el programa FRANJA y las comparamos con la prueba $t$ de Hutcheson (1970). Además, aplicamos el coeficiente de correlación de Spearman $\left(r_{s}\right)$ (Siegel \& Castellan 1988) para verificar si ambos método de análisis expresan un grado de asociación entre las presas consumidas. Adicional a ello, se aplicó el índice de Horn (Horn 1966) para evaluar el grado de similitud en la composición taxonómica (tipo de presas individuales) de presas presentes en estómagos y heces, utilizando el programa QUANTAN (Brower et al. 1977). Por último, se aplicó la prueba de Mann-Whitney (Siegel \& Castellan 1988, Wheather et al. 2011) para verificar diferencias en la abundancia de presas reflejada por ambos métodos de análisis.

\section{RESULTADOS}

Todas las presas identificadas en los estómagos y las heces de Bandurria y de Queltehue presentaron algún tipo de estructura corporal quitinosa indigerible, con excepción de las lombrices de tierra (Lumbricus spp.) que para su identificación en las heces requiere un método de cuantificación específico como el conteo de cerdas en preparaciones microscópicas teñidas con ácido pícrico (MacDonald 1983) (no efectuado en este trabajo debido a limitaciones logísticas).

En ambas especies de aves, los análisis estomacales revelaron 11 categorías de presas diferentes, mientras que en las heces sólo se detectaron ocho categorías de presas en la Bandurria y nueve en el Queltehue (Tabla 1). A pesar de ello, es evidente la correspondencia existente en el número de presas detectada por ambos métodos de análisis en las bandurrias $\left(r_{\mathrm{s}}=0,743 ; P=0,002 ; N=15 ; R^{2}=0,5520\right)$ (Fig. 
1). Para el caso del Queltehue la situación fue más aleatoria, con mayor dispersión de los valores $\left(r_{\mathrm{s}}=0,318 ; P=0,248\right.$; $N=15 ; R^{2}=0,1011$ ) (Fig. 2). Adicionalmente, los resultados no mostraron diferencias significativas en la abundancia de presas identificadas mediante ambos métodos, para la Bandurria (Mann-Whitney $U=79,00 ; P=0,174 ; N=30$ ) ni para el Queltehue (Mann-Whitney $U=78,50 ; P=0,161 ; N=$ $30)$, aunque se hallaron aproximadamente el doble de presas en los estómagos respecto a las heces en la Bandurria (831 vs. 460) y más del triple en el caso del Queltehue (82 vs. 26).

Al confrontar la diversidad y la varianza de los componentes dietarios obtenidos por ambos métodos de análisis, se evidenció que en bandurrias y queltehues, las diversidades de presas identificadas son mayores en los estómagos, mientras que las varianzas fueron mayores en los análisis fecales de ambas especies de aves (Tabla 2). Para la Bandurria, el índice de sobreposición de Horn reveló una alta similitud de presas en las dietas obtenidas por ambos métodos aplicados $\left(R_{0}=0,914\right)$. Por el contrario, en el caso del Queltehue, la similitud de presas en la dieta reflejada por ambos métodos es intermedia $\left(R_{0}=0,536\right)$. Aunque en este último caso la diversidad no difirió entre estómagos y heces, sí hubo diferencias en la composición taxonómica de presas en la dieta, es decir, ambos métodos reflejaron diferencias en el tipo presas individuales consumidas.

TABLA 1. Número total de presas consumidas por la Bandurria y el Queltehue en el mes de Agosto 1999 de acuerdo al método de análisis dietario aplicado. $(\mathrm{L})=$ estados larvales, $(\mathrm{a})=$ estados adultos. * Datos obtenidos de Gantz et al. (2009).

TABLE 1. Total prey number consumed by the Black-faced Ibis and Southern Lapwing during August 1999 in accordance with the method of dietary analysis applied. (L) = larval stages, (a) = adult stages. * Data obtained from Gantz et al. (2009).

\begin{tabular}{|c|c|c|c|c|}
\hline \multirow[b]{2}{*}{ Presas consumidas } & \multicolumn{2}{|c|}{ Bandurria } & \multicolumn{2}{|c|}{ Queltehue* } \\
\hline & $\begin{array}{c}\text { Estómagos } \\
(\mathrm{n}=10)\end{array}$ & $\begin{array}{c}\text { Heces } \\
(\mathrm{n}=45)\end{array}$ & $\begin{array}{l}\text { Estómagos* } \\
\quad(\mathrm{n}=10)\end{array}$ & $\begin{array}{c}\text { Heces } \\
(\mathrm{n}=34)\end{array}$ \\
\hline Agrotis spp. (L) & 131 & 23 & 49 & 3 \\
\hline Araneae (a) & 5 & 0 & 3 & 0 \\
\hline Carabidae (a) & 16 & 2 & 15 & 2 \\
\hline Ceroglosus chilensis (a) & 3 & 0 & 0 & 0 \\
\hline Ceriotus torulosus (a) & 3 & 0 & 0 & 0 \\
\hline Curculionidae (a) & 0 & 1 & 0 & 14 \\
\hline Curculionidae (L) & 10 & 3 & 19 & 2 \\
\hline Dalaca spp. (L) & 244 & 142 & 3 & 12 \\
\hline Diptera (L) & 0 & 0 & 2 & 0 \\
\hline Elateridae (L) & 5 & 4 & 14 & 6 \\
\hline Forficula auricularia (a) & 16 & 8 & 2 & 1 \\
\hline Hemiptera (a) & 0 & 0 & 3 & 0 \\
\hline Hylamorpha spp. (L) & 441 & 288 & 5 & 3 \\
\hline Lumbricus spp. & 126 & 0 & 5 & 0 \\
\hline Hymenoptera & 0 & 0 & 0 & 1 \\
\hline TOTAL & 1000 & 471 & 120 & 44 \\
\hline
\end{tabular}

TABLa 2. Diversidad $\left(H^{\prime}\right)$ y varianza $\left(s^{2}\right)$ de los contenidos de presas identificadas en estómagos y heces de la Bandurria y el Queltehue.

TABLE 2. Diversity $\left(H^{\prime}\right)$ and variance $\left(s^{2}\right)$ of the identified preys in the stomachs and feces of the Black-faced Ibis and Southern Lapwing.

\begin{tabular}{lcccc}
\hline & \multicolumn{2}{c}{ Estómagos } & \multicolumn{2}{c}{ Heces } \\
& Diversidad & Varianza & Diversidad & Varianza \\
\hline Bandurria & 1.499 & \pm 0.001 & 0.988 & \pm-0.002 \\
Queltehue & 1.846 & \pm 0.008 & 1.810 & \pm-0.014 \\
\hline
\end{tabular}




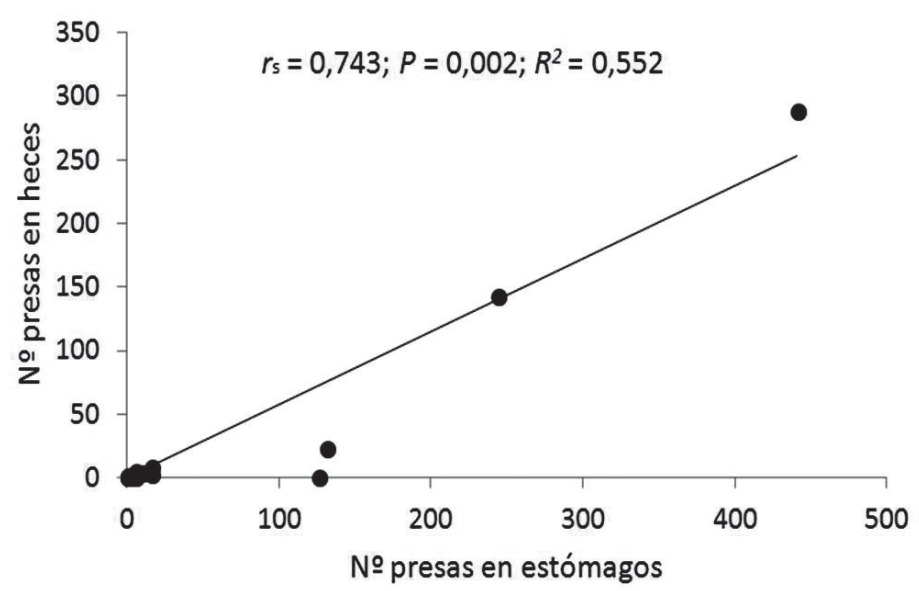

FiguRA 1. Grado de asociación en el número de presas encontrado en estómagos y heces de la Bandurria.

FIGURE 1. Association level of the prey number found in the stomachs and feces of the Black-faced Ibis.

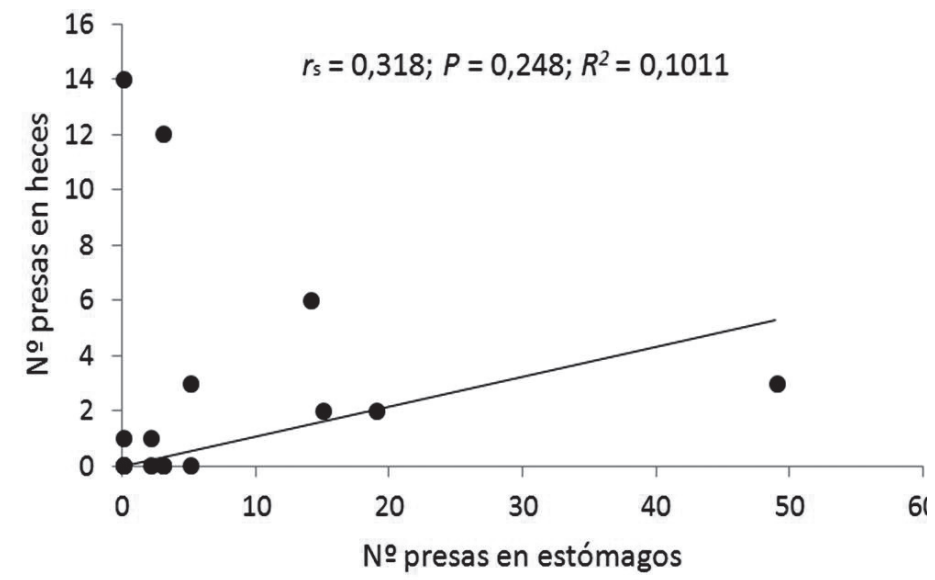

FIGURA 2. Grado de asociación en el número de presas encontrado en estómagos y heces del Queltehue.

Figure 2. Association level of the prey number found in the stomachs and feces of the Southern Lapwing.

\section{DISCUSIÓN}

De acuerdo a nuestros resultados, los estómagos lograron discriminar una dieta más completa que las heces. Adicionalmente, la riqueza y diversidad de presas estuvieron sub-representadas en los análisis fecales de ambas especies de ave. Estas diferencias probablemente sean consecuencia de una menor representación de las presas de cuerpos blandos como consecuencia de la digestión diferencial del contenido estomacal. Nuestros resultados son concordantes con la detección diferencial de ambos métodos de análisis dietarios señalado por diversos autores en numerosas especies de aves (Rosember \& Cooper 1990, Harris \& Wanless 1993, Votier et al. 2003, Barret et al. 2007).

Para el caso particular del Queltehue, sólo la abundancia de presas detectada en las heces fue coherente con la de los estómagos, sin embargo, los restantes parámetros dietarios como la riqueza, diversidad y la composición taxonómica de presas presente en las heces difirió marcadamente de las presas detectadas en los estómagos. El marcado sesgo observado entre ambos métodos de análisis dietario permiten sugerir que los análisis fecales en el Queltehue no serían confiables y podrían conducir a inferencias erróneas y a sesgos interpretativos inadecuados referente a la ecología trófica de esta especie de ave, en particular cuando se realizan análisis comparativos confrontando estudios basados en ambas metodologías de análisis. Para el caso de la Bandurria, si bien los análisis fecales mostraron una menor riqueza y diversidad de presas, también arrojaron una abundancia, composición taxonómica y una correlación de presas muy próxima a las detectadas en los estómagos, 
lo que sugiere que en esta especie de ave, los análisis fecales entregan resultados confiables y concordantes con los análisis de contenido estomacal. Sin embargo, en las interpretaciones de resultados con este método, se debe considerar la reducida habilidad de los análisis fecales para detectar el número de presas totales.

En consecuencia, para el caso particular de la Bandurria, nuestros resultados serían coherentes con lo señalado por la literatura, en cuanto a que los análisis fecales podrían constituir un método adecuado para evaluar proporciones de presas consumidas por aves insectívoras (Taylor \& O’Halloran 1997), a pesar de las limitaciones del método generadas por la digestibilidad diferencial de las presas (Rosemberg \& Cooper 1990, Barrett et al. 2007, Weiser \& Powell 2011). Por tanto, consideramos que los análisis fecales en la Bandurria, no obstante las restricciones inherentes al método, constituirían una alternativa factible de aplicar en estudios dietarios a escalas espaciales y temporales más extensas, obviamente teniendo en consideración que las presas de cuerpos blandos frecuentemente están subrepresentadas en análisis de este tipo (Jordan 2005).

Sin embargo, la riqueza reducida de presas detectada en los análisis fecales en la Bandurria podría limitar su uso en estudios centrados en evaluaciones de amplitud de nicho trófico. No obstante, esta limitante podría subsanarse con el uso complementario de análisis dietarios moleculares y fecales. Los análisis de isotopos estables (Bearhop et al. 2004) actualmente son utilizados extensivamente en estudios dietarios de aves (Barret et al. 2007, Vitz \& Rodewald 2012), esta técnica no está sesgada por la digestibilidad diferencial de las presas, pero en cambio, presenta la limitante de la inhabilidad de examinar la dieta a niveles taxonómicos finos (Weiser \& Powell 2011, Vitz \& Rodewald 2012) y en consecuencia, podría ser una herramienta integrativa poderosa para análisis de amplitud de nicho cuando se combina con análisis dietarios convencionales, como los análisis fecales, que proveen mayor información al nivel taxonómico de especie (Bearhop et al. 2004, Weiser \& Powell 2011). De esta forma, los análisis fecales, complementados con los de isótopos estables, podrían constituir una herramienta de análisis dietario confiable y factible de aplicar en estudios dietarios a largo plazo.

\section{AGRADECIMIENTOS}

Agradecemos a los propietarios de predios agrícolas por su gentileza de autorizar el acceso a su propiedad. Al Sr. Eladio Rojas del Laboratorio de Entomología del S.A.G. Osorno, por la identificación de los restos entomológicos. A la Dirección de Investigación de la Universidad de Los Lagos por el apoyo financiero del Proyecto de Investigación
$\mathrm{N}^{\circ} 3300$ del cual fueron obtenidos los datos para el presente estudio. A los revisores anónimos por sus invaluables contribuciones para mejorar el manuscrito

\section{BIBLIOGRAFÍA}

Barret, R.T., Camphuysen, K.C.J., Anker-Nielssen, T., Chardine, J.W., Furness, R.W., Garthe, S., Hüprop, O., Leopold, M.F., MonteVecchi, W.A. \& Veit, R.R. 2007. Diet studies of seabirds: a review and recommendations. ICES Journal of Marine Science 64:1675-1691.

Bearhop, S., Adams, C.E., Waldron, S., Fuller, R.A. \& Macleod, H. 2004. Determining trophic niche width: a novel approach using stable isotopes analysis. Journal of Animal Ecology 73:1007-1012.

Brower, J.E., Zar, J.H. \& von Ende, C.N. 1977. Field and laboratory methods for general ecology. $3^{\text {a }}$ Edición. Wm. C. Brown Publishers, Iowa, USA. 237 pp.

Brown, K.M. \& EwINS, P.J. 1996. Technique-dependent biases in determination of diet composition: an example with RingBilled Gulls. The Condor 98:34-41.

Calver, M.C. \& Wooler. R.D. 1982. A technique for assessing the taxa, length, dry weight and energy content of the arthropod prey of birds. Australian Wildlife Research 9:293-301.

Clare, E.L., Faser, E.E., Braid, H.E., Brock Fenton, M. \& Hebert, P.D.N. 2009. Species on the menú of a generalist predator, the Eastern red bat (Lasiurus borealis): using a molecular approach to detect arthropod prey. Molecular Ecology 18:2532-2542.

Gantz, A., SAde, S. \& Rau, J. 2009. Winter diet and feeding preferences of the Southern Lapwing (Vanellus chilensis, MOLINA 1782) in pastures of southern Chile. Boletín Chileno de Ornitología 15:87-93.

GonZÁLEZ, R.H. 1989. Insectos y ácaros de importancia agrícola y cuarentenaria en Chile. Ediciones Ograma S.A., Santiago, Chile. 310 pp.

HaRris, M.P. \& WanLess, S. 1993. The diet of Shags Phalacrocorax aristotelis during the chick-rearing period assessed by three methods. Birds Study 40:135-139.

Horn, H.S. 1966. Measurement of "overlap" in comparative ecological studies. American Naturalist 100:419-424.

Hutcheson, K. 1970. A test for comparing diversities based on the Shannon formula. Journal of Theoretical Biology 29:151154.

Jordan, M.J.R. 2005. Dietary analysis for mammals and birds: a review of field techniques and animal-management applications. International Zoo Yearbooks 39:108-116.

Korschgen, L.J. 1987. Procedimientos para análisis de hábitos alimentarios. En: Manual de técnicas de gestión de vida silvestre (Ed. Rodríguez, R.), pp. 119-134. 4ª Edición. Ediciones WWF. USA.

Macdonald, D.W. 1983. Predation on earthworms by terrestrial vertebrates. En: Earthworm Ecology. From Darwin to Vermiculture (Ed. Satchell, J.E.), pp. 393-414. Chapman and Hall, London.

Muñoz-Pedreros, A., Rau, J. \& Yañez, J. 2004. Aves rapaces de Chile. CEA Ediciones, Valdivia, Chile. 387 pp.

PeÑa, L.E. 1998. Introducción al estudio de los insectos de Chile. 
Editorial Universitaria, Santiago, Chile. 253 pp.

Rosemberg, K.V. \& Cooper, R.J. 1990. Approaches to avian diet analysis. Studies in Avian Biology 13:80-90.

Siegel, S. \& Castellan, N.J. 1988. Nonparametric statistics for the behavioral sciences. McGraw-Hill, New York. 399 pp.

TaYlor, A.J. \& O'Halloran, J. 1997. The diet of the Dipper Cinclus cinclus as represented by faecal and regurgitate pellets: a comparison. Bird Study 44:338-347.

Vitz, A.C. \& Rodewald, A.D. 2012. Using stable isotopes to investigate the dietary trophic level of fledgling songbirds. Journal of Field Ornithology 83:73-84.

Votier, S.C., Bearhop, S., MacCormick, A., Ratcliffe, N. \& Furness, R.W. 2003. Assessing the diet of great skuas, Catharacta skua, using five different techniques. Polar Biology 26:20-26.
Waldner, T. \& Traugott, M. 2012. DNA-based analysis of regurgitates: a noninvasive approach to examine the diet of invertebrate consumers. Molecular Ecology Resources 12:669-675.

Weiser, E.L. \& Powell, A.N. 2011. Evaluating gull diets: a comparison of conventional methods and stable isotope analysis. Journal of Field Ornithology 82:297-310.

Wheather, C.P., Bell, J.R. \& Cook, P.A. 2011. Practical Field Ecology. A Project Guide. John Wiley \& Sons, Oxford, UK. 362 pp.

Wobeser, G.A., Spraker, T.R. \& Harms, V.L. 1987. Colección y preservación de materiales biológicos en el campo. En: Manual de técnicas de gestión de vida silvestre (Ed. Rodríguez, R.), pp. 563-577. 4ª Edición. Ediciones WWF. USA.

Recibido: 09.09.15

Aceptado: 25.04.16 